\title{
Equipment for the study of operating processes in braille reading
}

\author{
A. NOBLET, H. RIDELAIRE, and G. SYLIN \\ Université Libre de Bruxelles, Bruxelles, Belgique
}

\begin{abstract}
The device described in this paper measures the spatial coordinates of the fingers of blind readers decoding texts written in braille. A light-emitting diode is fixed onto each of the two reading fingers, and a television camera with a MOS pickup device delivers a signal from which is extracted the information required to deduce the vertical and horizontal coordinates of the fingers. The results of these measurements appear as eight-digit binary numbers available for real-time computer data processing.
\end{abstract}

To improve our understanding of the reading processes of the blind, Bertelson and Mousty (1982) studied the operating modes used by braille readers. The method consists of recording on a videotape the hands of blind readers and in analyzing these recordings to study the finger movements over the page. Preliminary results indicate that the patterns of two-handed exploration are one result of two different principles of hand exploration: conjoint and disjoint (Mousty, Bertelson, \& Hublet, 1981). With disjoint exploration, it appears that the two fingers explore, sometimes simultaneously, different passages. This overlapping is one of the subjects of our research. By adding measurements of time to those of spatial position, it is possible to calculate other parameters, such as speed and acceleration of the fingers. This provides more detailed information on the various components of total reading time.

In this paper, we describe computer-controlled equipment for recording the movements of the fingers over a page of braille. The system allows for real-time data acquisition and fast data processing. Other tracking devices used during braille reading employ either kimographic recordings made by attaching styli to the fingers of the reader (Kusajima, 1974) or video recordings (Davidson, Wiles-Kettenmann, Haber, \& Appelle, 1980). Unfortunately, these studies either do not provide detailed descriptions of the systems, or, if they do, the information could induce unusual reading modes.

\section{MEASUREMENT PRINCIPLE}

A light source is fixed on the reading fingers, generally the forefingers, and their location on the page is deduced from the video composite signal delivered by a TV camera. The horizontal and vertical coordinates of

This work has been realized in collaboration with the Laboratoire de Psychologie Expérimentale. Thanks are due P. Bertelson, head of this department, and P. Mousty for their support during its development. The authors' mailing address is: Laboratoire de Physique Electronique, Université Libre de Bruxelles, Bruxelles, Belgique. each finger are measured every $40 \mathrm{msec}$ and are available either in analog or digital form for direct computer treatment. The light sources fixed on the reading fingers are subminiature light-emitting diodes (LEDs).

Discrimination between the two fingers is obtained by synchronizing the lighting of the diodes with the frame TV pulses of the camera. Each diode is switched "on" during the analysis of half a TV image so that the spatial coordinates are available every $20 \mathrm{msec}$, alternately for one finger and then the other (Figure 1). The measurement of the vertical coordinate can be easily obtained by counting the number of TV lines scanned from the appearance of the video pulse (generated by the solid-state lamp) to the following frame pulse. The horizontal position of the fingers is also deduced by counting pulses generated by a local oscillator. Their number is proportional to the time interval between the horizontal (line) synchronization pulse and the video signal located on the same line (Figure 1). This interval is directly related to the finger position on the text line. This method has the advantage of delivering digital information in a form directly available for computer data processing.

The use of digital-to-analog converters makes it possible to reproduce the variation of the coordinates with time, or the path of the fingers on the page, using $x-t$ or $x-y$ recorders.

A previous analysis made with two cameras, one above the hands and the other to verify the fingers actually in contact with the text, showed that, generally, reading is accomplished with the tips of the index fingers (Bertelson, Mousty, \& D'Alimonte, in press). As a result, we decided to use only one camera, located vertically above the reading page; in the analysis of exploration movements, it was assumed that the forefingers were used to read the braille. It is possible to fix the light sources on two fingers of the same hand and to obtain information on the movements of each finger in the reading process, if this is necessary.

Since we are not interested in the micromovements of the fingers, a time resolution of $40 \mathrm{msec}$ is sufficient. Note, however, that by fixing the two LEDs on the same 


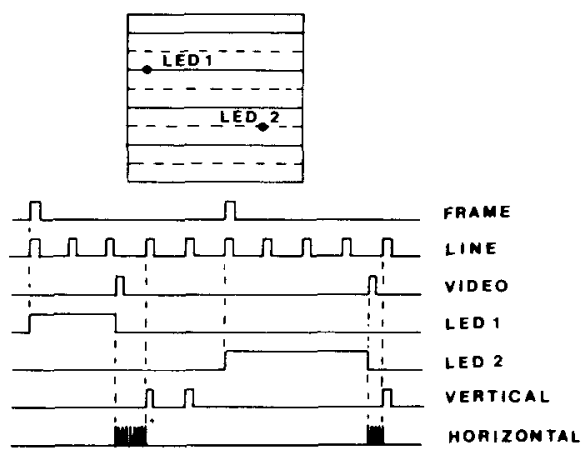

Figure 1. Principle of the measurement.

finger, time resolution can be halved in the case of onehand reading.

\section{CHARACTERISTICS OF THE BRAILLE CELL}

A braille cell is a matrix of $2 \times 3$ dots with, in our case, the following mean characteristics: diameter of the dots $=1.52 \mathrm{~mm}$; height of the dots $=0.43 \mathrm{~mm}$; interdot distance $=2.28 \mathrm{~mm}$. Each cell has a width of $3.8 \mathrm{~mm}$ and a length of $6.08 \mathrm{~mm}$; the intercell distance is $4.07 \mathrm{~mm}$. On the standard text page used, there are 40 cells and intervals on a line and 14 lines per page. The interline distance is $5.59 \mathrm{~mm}$.

\section{RESOLUTION OF THE MEASUREMENT}

The optical system is devised in such a way that the standard text page $(255 \times 145 \mathrm{~mm})$ occupies approximately the entire width of the TV image. The pulse frequency, allowing the location of the finger on a line, is $3.9 \mathrm{MHz}$; we dispose, then, of \pm 200 pulses for the effective duration of a TV line $(\sim 52 \mu \mathrm{sec})$. Since there are 40 characters per line, this leads to a horizontal resolution of about 0.2 characters per pulse, which means approximately $1.2 \mathrm{~mm}$.

Two successive measurements, for a same finger, are separated by $40 \mathrm{msec}$. It takes a good reader approximately $3 \mathrm{sec}$ to read a complete text line, which implies that the maximum finger displacement between two measurements is about .5 characters, or $3 \mathrm{~mm}$. In the vertical direction, the text page occupies $3 / 5$ of the TV image. This corresponds to $\pm 200 \mathrm{TV}$ lines ( 312.5 lines in a frame for the European standard) and, since there are 14 text lines on a page, leads to a vertical resolution of less than 0.1 characters for a TV line, or $1 \mathrm{~mm}$.

\section{CHOICE OF THE TV CAMERA}

The TV camera must provide, with great reliability, the variation of the light-source positions, from one frame to the other. Therefore, a TV camera with very low lag is necessary; this explains the choice of a solid-state pickup device. This type of camera is free of geometric distor- tion and is compact, lightweight, and highly sensitive. The selected camera (Hitachi KP-120) has a horizontal resolution of more than 240 lines at the center and a vertical resolution of more than 190 lines at the center. This is fully compatible with the previously described characteristics. Its spectral response curve shows a relative sensitivity of $75 \%$ at the wavelength of the red light emitted by the diodes. The minimum prescribed illumination of this camera is $10 \mathrm{~lx}$ for a lens aperture of $\mathrm{f} 1.4$. Knowing the luminous intensity of the diode, one can calculate that the illumination, integrated over the entire text page, will be about $200 \mathrm{~lx}$, the camera being located $1 \mathrm{~m}$ above the page. This is more than enough to obtain a diode video signal quite distinct from the noise generated by the illumination of the text page.

\section{DESCRIPTION OF THE ELECTRONIC CIRCUIT}

Figure 2 shows a simplified block diagram of the electronic circuit. On the one hand, the vertical synchronizing pulse (frame) $V$ triggers the flip-flop FFI, which sets FF2. The output of gate P1 goes on and allows the lighting of the solid-state diode LED1. A video signal D, detected in the analyzed frame, resets FF2 and turns LED1 off. This signal also sets FF4 and opens the gate P2, allowing the horizontal synchronizing pulses (line) $\mathrm{H}$ to be counted until the appearance of the following frame pulse, which resets FF4 and thus closes P2. After a delay RET, this pulse triggers the monostable MT1 and allows the content of counter 1 to be transferred in register 1 . The counter is then zeroed by triggering the monostable MT2.

On the other hand, gate P3 is normally open ( $\bar{Q}$ FF3 $=1$ ) and counter 2 receives the pulses from generator $G$. Each line pulse $\mathrm{H}$ resets this counter to zero. The detec-

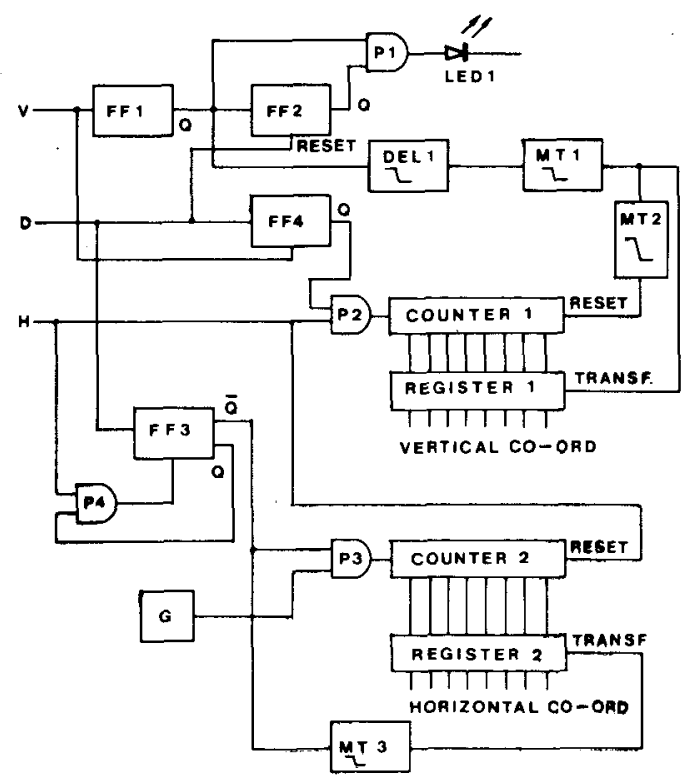

Figure 2. Simplified block diagram. 


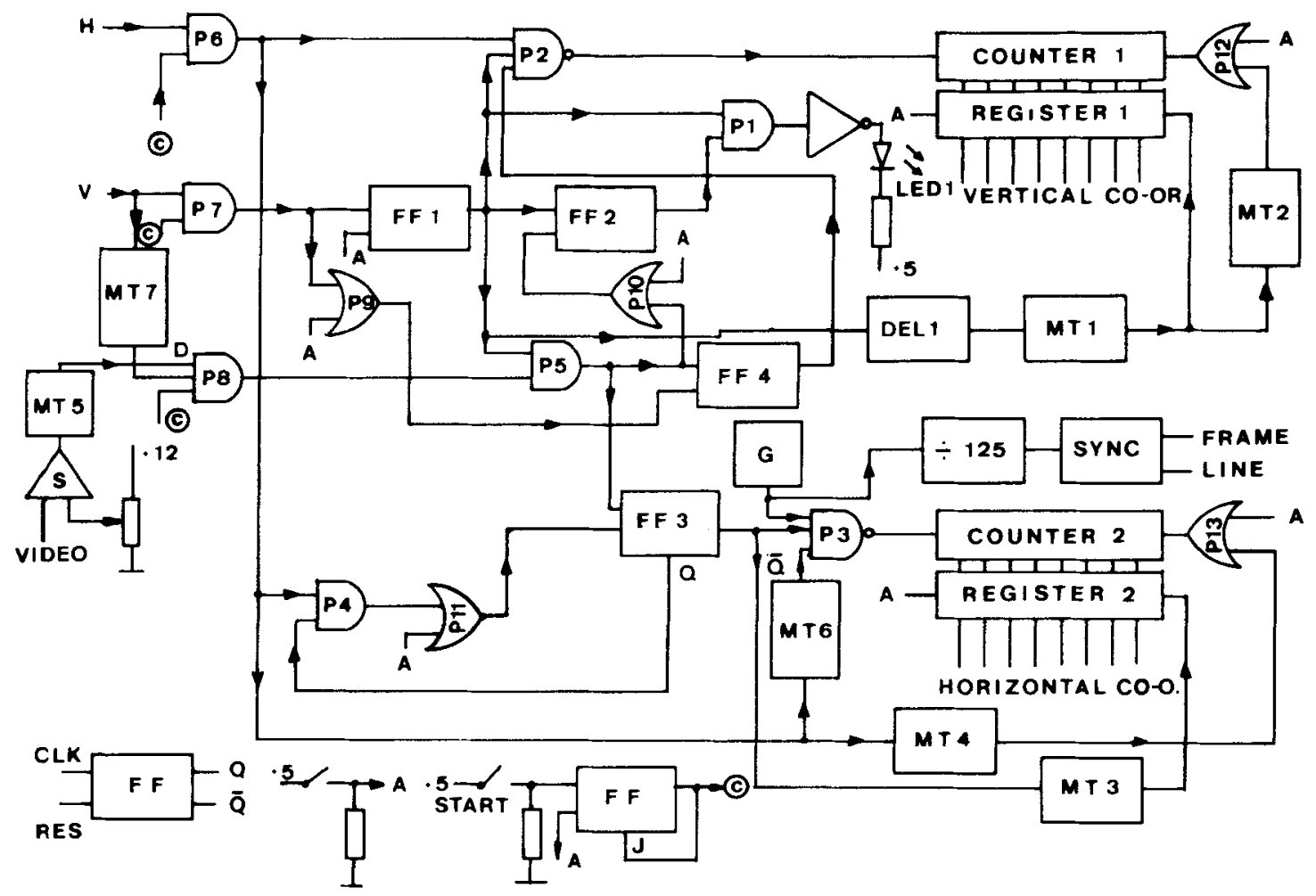

Figure 3. Detailed block diagram.

tion of a video pulse sets FF3 and thus stops the counting of the generator pulses by closing gate P3. At the same time, the monostable MT3 sends a transfer pulse to register 2 . Hence, the output of this register shows a content that indicates the horizontal finger position on the text line. At the end of the TV line, the synchronizing pulse $\mathbf{H}$ resets FF3, through gate $\mathrm{P} 4$, and also resets counter 2 . So, for each TV frame, the contents of the two registers are eight-digit binary numbers whose values are proportional to the vertical and horizontal coordinates, the origin being located in the lower left corner of the TV image.

The more detailed block diagram of Figure 3 shows that gate P5 allows the video signal D to be transmitted only during the frame analyzed when the diode LEDl is switched "on." This is necessary because there is another circuit, similar to the one described, that takes care of the second reading finger. Therefore, the video signals from the two LEDs must be directed to the right circuit.

The functioning principle of the solid-state camera makes it necessary to turn the diode off immediately after the detection of a video signal. Otherwise, a second pulse, from the same video signal, will be generated during the analysis of the following frame. The target of the camera is a two-dimensional photosensor array, and the electric charge accumulated in a sensitive picture element (pixel) grows as long as this element is illuminated. When a reading is made, the pixel discharges and gives rise to a video pulse whose amplitude depends on the quantity of light received. Therefore, if the LED is not turned off immediately after a reading, the pixel, still illuminated, will get a new charge, which remains constant once the light source goes off. During the analysis of the following frame, another pulse is then generated, originating from the same information as that of the preceding frame. Figure 4 shows that this second pulse can be of even higher amplitude than the first, when the diode is located at the top of the page.

The detailed block diagram (Figure 3 ) shows other circuit improvements. At the beginning of each TV line $\mathrm{H}$, the triggering of monostable MT6 closes gate P3 for a given time interval and prevents counting of the genera-

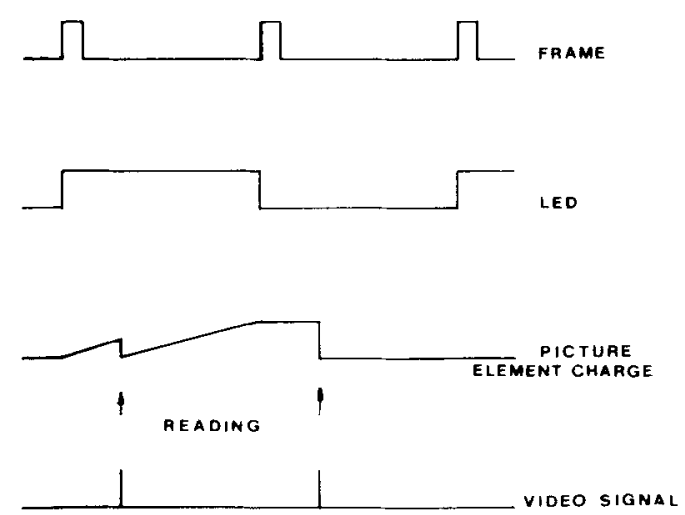

Figure 4. False reproduction of a measurement. 
First Finger Section
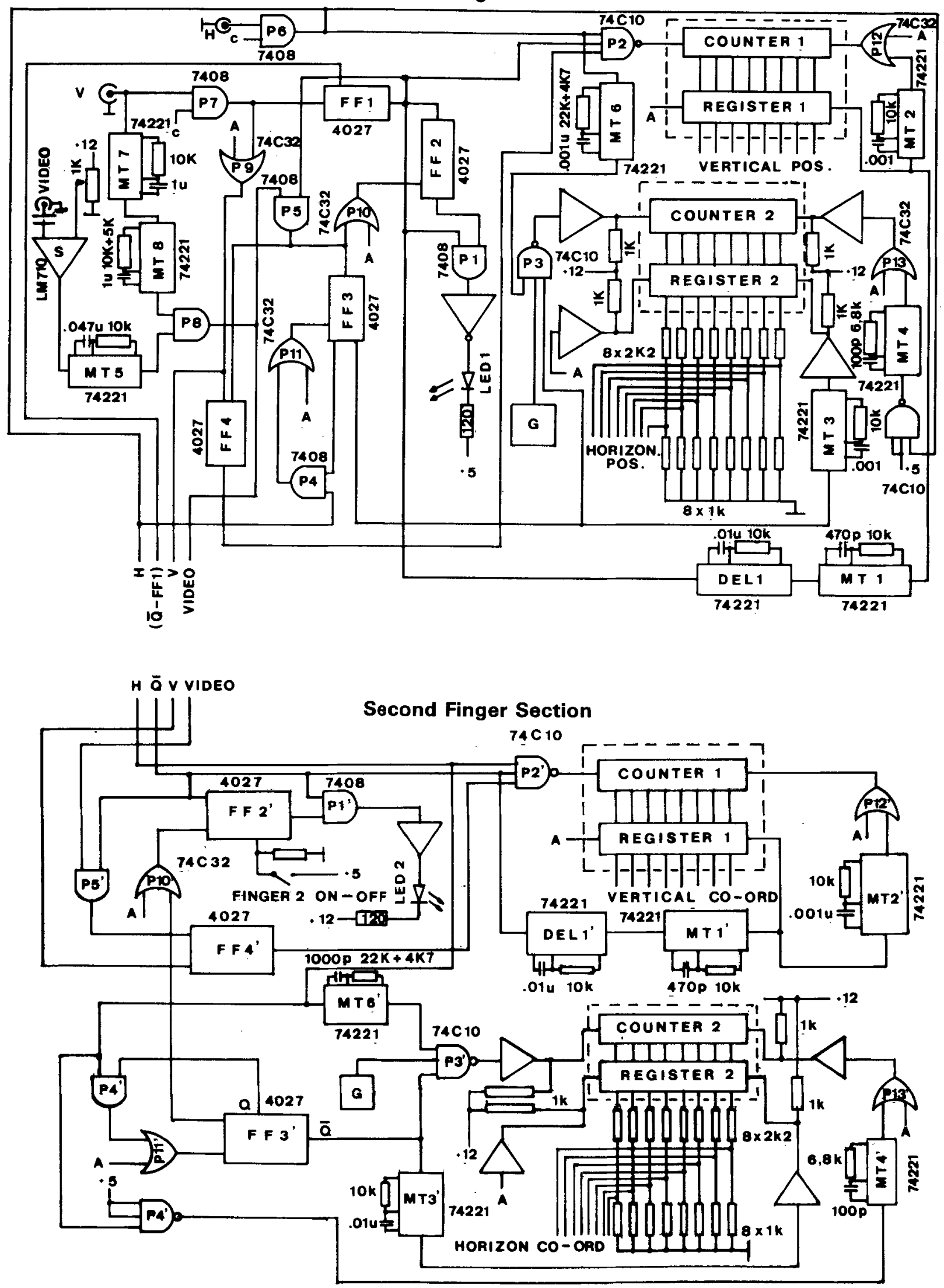

Figure 5. Complete electronic circuit. 
tor pulses during this closing time. To set this time, the diode is placed at the beginning of the page and the output pulse width of MT6 is adjusted in order to have a null output at register 2 , so the horizontal axis origin coincides with the beginning of the text line. A threshold circuit $S$ eliminates noise from the video signal. This circuit feeds the shaping monostable MT5.

Before a measurement can be initiated, the various flipflops and counters must be reset. This is provided through the push-button STOP and the OR gates P9 through P13. The start of the measurement is activated through pushbutton START and gates P6, P7, and P8.

The TV camera is synchronized by the generator $G$ : After a division by 125 , the output signal of this quartzcontrolled oscillator feeds a synchronizing circuit whose outputs deliver the horizontal and vertical synchronizing pulses to the camera after adequate impedance matching.

Another circuit, very similar to the one just described, measures the coordinates of the second reading finger. LED2 is fed by the $\bar{Q}$ output of FF1. Thus, a measurement for this finger is taken during the frame following those during which the first finger position has been measured. Figure 5 shows the complete circuit. The timing of a measurement for each finger is given in Figure 6.

\section{DEVICE CONSTRUCTION}

In order to reduce power consumption, MOS-type integrated circuits are used. The various components implied in the determination of delays or pulse shapes are selected for good temperature stability.

The horizontal position counters have a $12-\mathrm{V}$ power

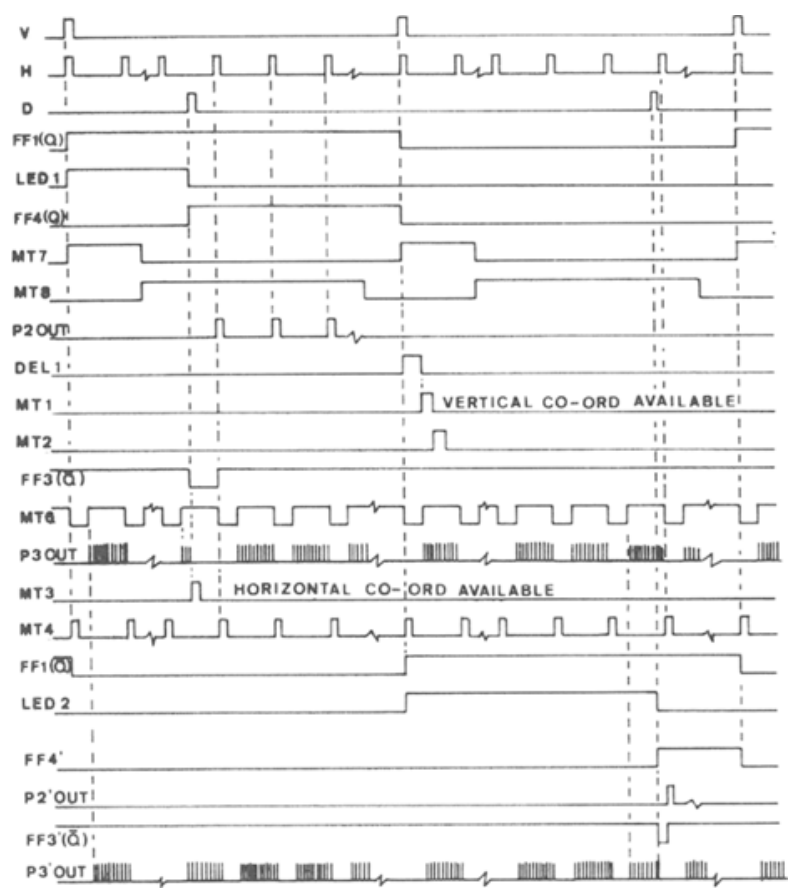

Figure 6. Fiming diagram of the measurement. supply. This increases their bandwidth, which, under $5 \mathrm{~V}$, is limited to $4 \mathrm{MHz}$. It is therefore necessary to include open-collector gates to match the signal levels, the output signals being adapted to TTL level by resistive dividers, as can be seen in Figure 5.

The quartz-stabilized clock oscillator has a frequency of $3.90625 \mathrm{MHz}$. It is enclosed in an earthed metal can to avoid radiations. The printed circuit boards are plugged into a chassis together with the power supplies. A front panel switch allows the user to inhibit the measurement of finger 2 (switch DGT2 in Figure 5).

The TV camera is fastened on a support moving along a vertical axis. A pulley-counterweight device makes the adjustment of the camera easier. With a standard text page, the target of the camera, equipped with a $12.5-\mathrm{mm}$ focal length lens, is at approximately $50 \mathrm{~cm}$ above the reading table. The LEDS are soldered onto little disks of printed circuit, which are fixed onto the nails of the reader with double-faced adhesive tape. Two very light and flexible wires connect this diode to a miniature jack plugging in a connector mounted on a wristwatch strap.

The measuring device is interfaced with a microcomputer Apple II by two adapter circuits VIA SY6522. Each of these circuits is able to handle two eight-digit binary numbers. The vertical counter resetting pulse sends an interrupt order to the VIA circuit, and the interrupt service routine reads and stores the information from the input registers. This allows real-time measurements, the time information being immediately available due to the periodic character of these measurements. If the analysis is to be made from a video recording (Mousty et al., 1981), it is necessary to add a time reference. Therefore, an electronic chronometer is located on the reading table, above the text page. Since this unwanted light source appears in the upper part of the TV image, it is necessary to cancel the measurements in this area. This is achieved by adding monostable MT7 (Figure 3), which, when triggered by the frame pulse, closes gate P8 during the analysis of this part of the image. We have found that for a lens aperture of $f \mathbf{8}$, the video signal saturates for a uniform illumination of the text page of $800 \mathrm{~lx}$, when the camera is located $50 \mathrm{~cm}$ above the reading table. This verifies the result previously stated $(\sim 200 \mathrm{~lx}$ at $1 \mathrm{~m})$. For a lens aperture of $\mathrm{f} 5.6$ and the threshold circuit set at $0.5 \mathrm{~V}$, the device is able to discriminate the diode signal for a page illumination varying from 0 to $4001 x$.

The selected solid-state camera, internally synchronized, functions at the American standard, 60 frames/sec, 525 lines/image. In our application, it is externally synchronized and functions at the European standard, $50 \mathrm{frames} / \mathrm{sec}, 625$ lines/image, but the image still contains $\mathbf{5 2 5}$ useful lines. There is therefore a loss of $\mathbf{5 0}$ lines in the lower part of each frame; because the text page is completely included in the analyzed image, this is not a drawback.

Earlier, we noted the presence of a digital chronometter in the upper part of the image. Because measurement is inhibited in this area, there is a further loss of 75 lines, 
and, finally, the vertical synchronizing pulse occupies 7 lines. This implies that there are 180 useful lines for the text page. This has been verified from measurements taken for the lowermost and uppermost position of the diode on the page, 57 and 237, respectively: The vertical resolution is therefore 0.08 characters/TV line, or $0.8 \mathrm{~mm}$, as expected. A similar agreement with our forecasts is found in the horizontal resolution measurement: For the leftmost and rightmost position of the LED, the values are 0 and 191, respectively. Thus, the measured resolution is 0.2 characters per pulse, or $1 \mathrm{~mm}$.

The linearity of the measurements is very good. Figure 7 presents the horizontal and vertical diode position measured by the device versus the length $\ell$ measured directly on the page. From the obtained results, it follows that the maximum error of linearity is $1 \%$ in the horizontal direction and $1.5 \%$ in the vertical.

Since we can verify that, while reading, the fingers do not move in such a way that the light beam remains in the same direction at all times, we determine the maximum allowable angle between an inclined light beam and the axis perpendicular to the page. The result of this measurement is, of course, related to the LED luminosity, which varies from one diode to the other. However, several measurements made on a sample of five diodes show that an angle of $\pm 50^{\circ}$ can be allowed without modifying the measured coordinates.

A complete test of the system was done with the aid of a good blind reader using two fingers. This subject reads 12 pages in a total time of about $10 \mathrm{~min}$. Therefore, a set of approximately 60,000 data was taken, and a detailed analysis of the results found only one to be false.

The same measurements were tested with a video recorder as the video source, instead of the TV camera. During these tests, we quickly realized that, with our recorder, the reading heads commutation gave rise to an additional video signal that appeared just before the frame pulse. These signals, interpreted by the device, systematically falsified the measurements. To remove this drawback, we added another monostable MT8 (Figure 5). The MT8 is triggered on the trailing edge of the inhibiting pulse from MT7 at the beginning of the frame, and opens gate $\mathbf{P 8}$ for $13 \mathrm{msec}$. The measurements also become inhibited in the lower part of the image, where the commutation signal appears. This is not inconvenient since this area includes the 50 lines that are not analyzed by the camera. False measurements are, however, more frequent in this mode of operation, because of the video tape dropout. Moreover, since the diode video pulses are very narrow, about $200 \mathrm{nsec}$, they are strongly attenuated by

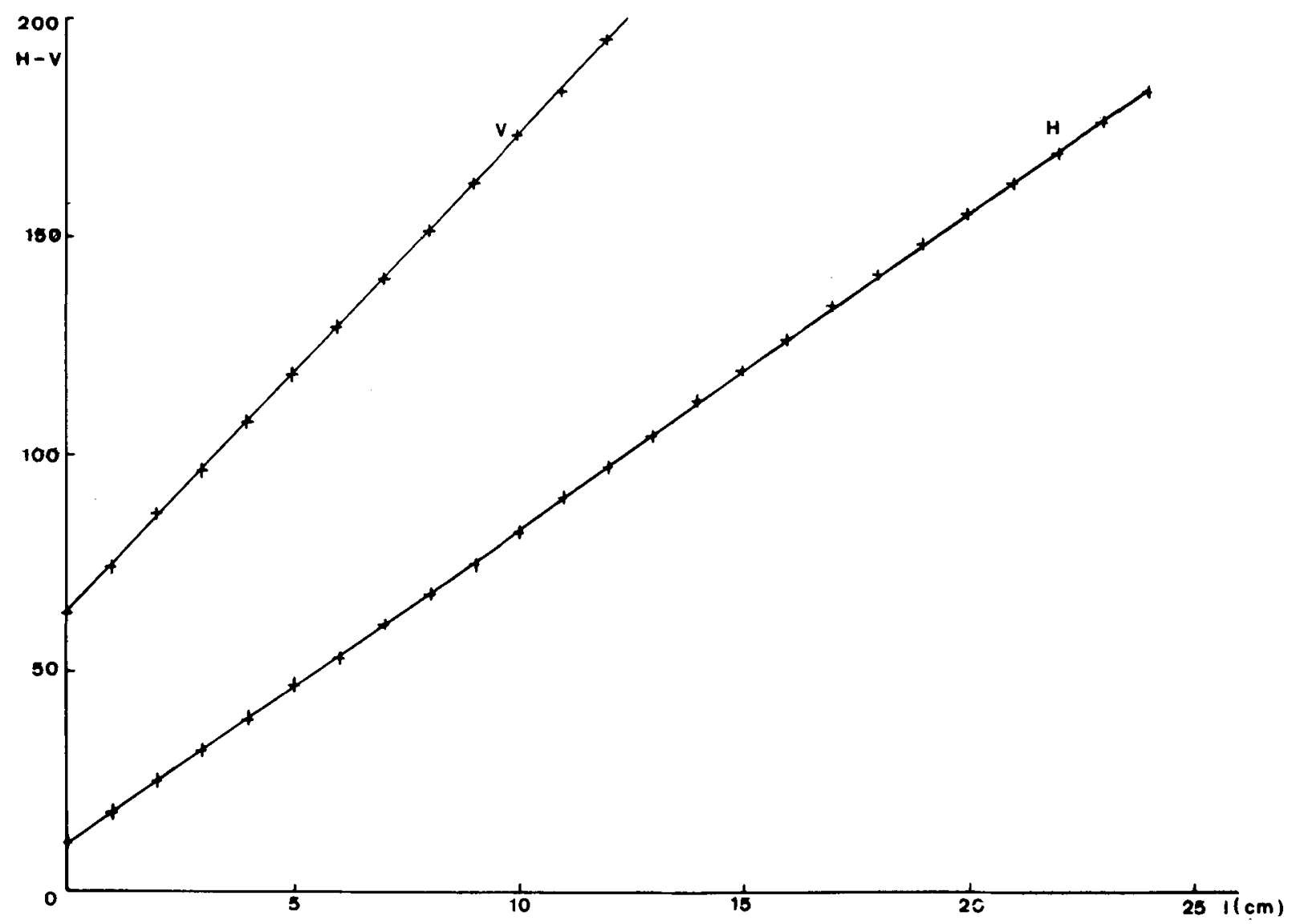

Figure 7. Linearity of the measurements. 
the recording process because of the limited bandwidth of the recorder. It is therefore necessary to include a video amplifier between the recorder and the measuring device, which in turn makes the system more sensitive to stray video signals. A software solution to this problem can be found by implementing a test set in the interrupt-handling routine.

The device is easily transportable. A light-dependent resistor luxmeter has also been built in order to measure and adjust the illumination of the page in order to ensure a good signal-to-noise ratio during the measurements. The stability and reliability tests show that no deviation is noticeable during $\mathbf{8 h}$ of functioning time and that the same measurement produces the same results over several days.

To improve the quality of the measurements made in the video recorder mode, an antidropout device can be added, but this is a sophisticated and expensive solution that is not justified, owing to the facility of the real-time measurements and to the powerful software solutions.

\section{REFERENCES}

Bertelson, P., \& Mousty, P. (1982). Modes opératoires dans la lecture de l'écriture braille. In J. Leplat \& P. Bertelson (Eds.), Hommage à J. M. Faverge. Le Travail Humain, 45, 13-23.

Bertelson, P., Mousty, P., \& D'Alimonte, G. (in press). A study of braille reading: 2) Patterns of hand activity in one-handed and twohanded reading. Quarterly Journal of Experimental Psychology.

Davidson, P. W., Wiles-Kettenmann, M., Haber, R. N., \& APPELLE, S. (1980). Relationship between hand movements, reading competence and passage difficulty in braille reading. Neuropsychologica, $18,629-655$.

KusAJima, T. (1974). Visual reading and braille reading: An experimental investigation of the physiology and psychology of visual and tactual reading. New York: American Foundation for the Blind.

Mousty, P., Bertelson, P., \& Hublet, C. (1981). Les rôles respectifs des mains dans la lecture du braille: Une étude exploratoire. In A. Harrisson-Covello, H., Herren, G. C., Lairy, P., Oleron, P., \& F. Robaye (Eds.), Les enfants handicapes (pp. 65-67). Paris: Presses Universitaires de France.

(Manuscript received July 20, 1984; revision accepted for publicaton January $25,1985$. 